\title{
Genetic Algorithm based Energy Aware Clustering Techniques: A Survey
}

\author{
Nivedita B. Nimbalkar \\ Department Of Computer \\ Engineering. \\ $\mathrm{KJ}$ College Of Engineering and \\ Management Research, \\ Pune .India
}

\author{
Soumitra S Das \\ Department Of Computer \\ Engineering , \\ Sathyabama University, \\ Chennai, India
}

\begin{abstract}
Wireless sensor networks are comprised of sensor nodes. Responsibility of the sensor nodes is to sense the atmospheric parameters and forward this data to cluster head. As the nodes are densely deployed, there is a possibility that the data is redundant. So in order to remove the redundancy, cluster head performs aggregation of the data and forward this data to base station. Sensor networks have a limitation that they are limited in energy. They are mostly deployed in harsh areas where it is hard to replace their batteries. One way of achieving energy efficiency is to use clustering technique. In clustering, clusters of sensor nodes are formed and every cluster has one cluster head. This paper discusses genetic algorithm based cluster formation techniques along with their merits and demerits.
\end{abstract}

\section{Keywords}

Wireless Sensor Networks (WSN), Cluster Head(CH) , Genetic Algorithm(GA),Clustering, Energy efficiency

\section{INTRODUCTION}

Wireless sensor networks are used in different applications like medical, agriculture, industry, military etc. They are mainly comprised of sensor nodes which are low in energy. As they are deployed in remote areas and it is hard to replace their batteries. Basic function of these sensor nodes is to sense environmental data. In clustering, sensor nodes are divided into clusters. Every cluster has a cluster head. All the sensor nodes sense the data and forward this data to Cluster Head $(\mathrm{CH})$. This data can be redundant so $\mathrm{CH}$ performs aggregation of this data and forward this data to Base Station (BS).Energy consumption is reduced in clustering because of reduction in communication distance [10]. Different technologies used for cluster head selection are genetic algorithm, fuzzy logic and neural networks. Paper discusses different genetic algorithm based cluster formation techniques.

Rest of the paper is organized as- second section discusses genetic algorithm and different genetic operators, third section discusses previous works on genetic algorithm based clustering techniques, their merits and demerits. Fourth section discuses conclusion and gives a future direction.

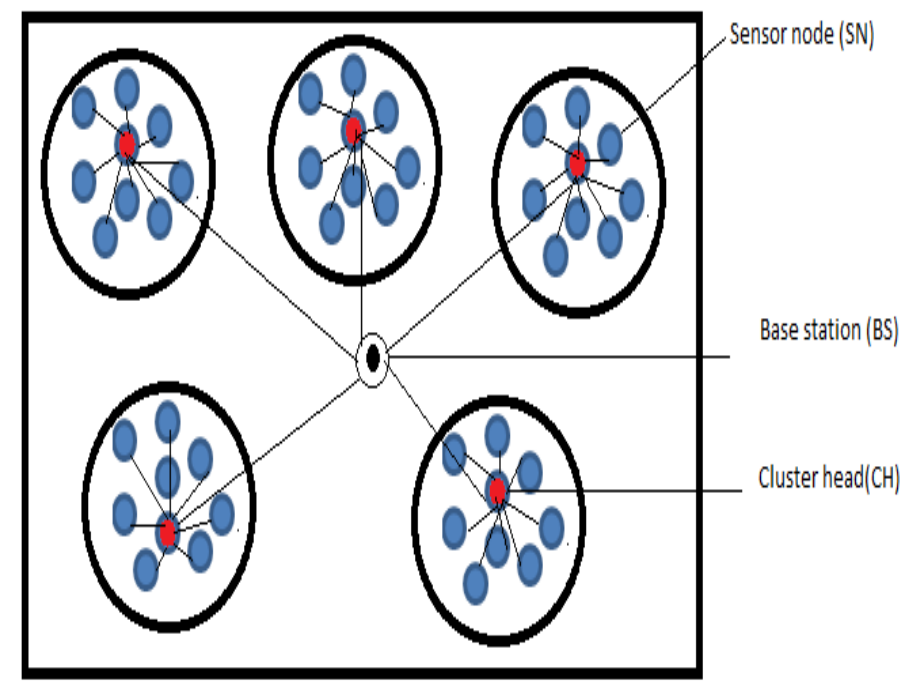

Fig. 1: Clustering in wireless sensor networks

\section{GENETIC ALGORITHM}

Genetic algorithm is a search algorithm. The concept of genetic algorithm was derived from Darwin's theory of evolution [9]. Darwin suggested that the individual who is fittest will survive in the competition of existence [8] and others will die out. Similar idea is used by genetic algorithm based cluster head selection. Here a fittest individual is a candidate for cluster head selection.

\subsection{Genetic Operators}

\subsubsection{Crossover}

In crossover, two parents are selected based upon the fitness. They are used to reproduce a new offspring. It is assumed that the offspring will inherit the good characteristics of both the parents and will have better characteristics than its parents. Most commonly used crossover is one point crossover. Here a random crossover point is selected and bits of parent chromosomes are swapped at that point to create a new offspring $[2,3,9]$.

Example-

Before crossover

Parent 1: 100010|010

Parent 2: 001000|110

After crossover

Offspring 1: 100010|110 


\section{Offspring 2: 001000|010}

\subsubsection{Mutation}

In mutation a bit which is ' 0 ' becomes ' 1 ' and the bit which is ' 1 ' becomes ' 0 '. Mutation is applied at mutation probability. After mutation, regular node may become a cluster head and a cluster head may become a regular node. When a $\mathrm{CH}$ becomes a regular node, members of that cluster join nearest cluster head and if a regular node becomes cluster head, the nodes which are close to it will join this cluster $[4,5]$.

\subsubsection{Selection}

Selection is used to select chromosomes for mating process i.e. crossover. Mating process will reproduce new chromosomes which will join current population. This will be called as a new generation.

\subsubsection{Fitness Function}

This is the function of various fitness parameters. This is used to evaluate the fitness of chromosomes. Fittest chromosomes will be used to create new population.

\section{RELATED WORK}

\subsection{Method 1}

In paper [4], Selim Bayrakli has proposed a cluster based method which is similar to LEACH. Method works in two phases: set up phase and steady state phase. Number of clusters and numbers of cluster heads are same because only one $\mathrm{CH}$ is associated with every cluster. Member nodes choose to join nearest $\mathrm{CH}$. In second phase, data is actually transmitted and for transmission of data to $\mathrm{CH}$, TDMA is used. Member nodes sense atmospheric data and send it to $\mathrm{CH} . \mathrm{CH}$ then sends this data in a packet to BS. This is considered as one round. Clusters are not changed after every round. Energy of the $\mathrm{CH}$ is checked and if found less than average energy of all the member nodes then $\mathrm{CH}$ is changed. Then the node who has highest residual energy becomes a $\mathrm{CH}$. The node which was $\mathrm{CH}$ in last round becomes a member node. Chromosome representation is used for the network. $\mathrm{CH}$ is represented using ' 1 ' and member node is represented using ' 0 '. Initial population is generated randomly. Every node is evaluated for fitness. Based upon fitness, fittest chromosome is selected for applying crossover and mutation.

Parameters used by paper [4] for fitness function: Cluster distance $\mathrm{C}$ ( It is sum of distance from member nodes to $\mathrm{CH}$ and $\mathrm{CH}$ to $\mathrm{BS})$, Round which first node $\operatorname{dies}\left(\mathrm{R}_{\mathrm{fnd}}\right)$,round which last node dies $\left(\mathrm{R}_{\text {lnd }}\right)$.

Fitness function as stated by [4]:

"Fitness function: $\sum \mathrm{i}$ ( fi X wi) $\forall$ fi $\in\left(\mathrm{R}_{\text {fnd }}, \mathrm{R}_{\text {lnd, }}-\mathrm{C}\right)$ "....(1)

\section{Advantages}

- Proposed method is better than classical LEACH in number of alive nodes.

- $\quad$ Lifetime of the network is enhanced.

- Cluster formation and cluster head selection using this method is energy efficient.

\section{Disadvantages}

- Residual energy is not a fitness parameter.
- Only rotation of the cluster heads takes place. Clusters are not changed throughout the lifetime of the network.

\subsection{Method 2}

In paper [5], basic aim is to determine clustering, position of cluster heads and optimize the energy consumption. Here also network is represented as bits of chromosome i.e. ' 0 ' represents a member node and ' 1 ' represents a $\mathrm{CH}$. Various genetic operators used in proposed method are: selection, mutation, fitness function and crossover.

Fitness function as stated by [5]:

“ Fitness $=\mathrm{RE}+((\mathrm{X} * \mathrm{SE})+(1-\mathrm{X}) *(\mathrm{~N}-\mathrm{CH})) ” \quad \ldots$

Here,

N-Total number of nodes

RE-Sum of residual energy in cluster heads

$\mathrm{CH}$-Total number of cluster heads

$\mathrm{X}$ - Weight factor whose value lies between 0 and 1

SE as stated by [5]:

“SE $=\sum_{\mathrm{i}=1}^{\mathrm{M}}\left(\mathrm{RS}_{\mathrm{i}}-\left(\mathrm{RH}_{\mathrm{i}}+\mathrm{HS}\right)\right)$ "

$\mathrm{RSi}$-Sum of energy which is required to transmit one message from all regular nodes to sink

RHi-Sum of the energy which is required to transmit one message from regular nodes to $\mathrm{CH}$.

HS- Sum of the energy which is required to transmit one message from all $\mathrm{CH}$ to sink

Simulation is done in Matlab. Proposed system was compared with classical LEACH. It was found that proposed system is better in following parameters:

- Uniform distribution of cluster heads in a network

- Total residual energy

- $\quad$ Alive nodes.

\section{Advantages}

- Uniform distribution of cluster heads is achieved.

- Lifetime of the network is enhanced by consuming energy uniformly.

- Numbers of alive nodes are more in proposed method in different rounds as compared to classical LEACH.

\section{Disadvantages}

- A single parameter i.e. energy is considered in a fitness function.

- Distance of the node from sink is not a fitness calculation parameter.

- Also centrality of the node and mobility of the node are not considered.

\subsection{Method 3}

In paper [6], Abbas Karimi has proposed a method. Fitness function calculation depends upon difference of energy of chromosomes in current and previous round. Chromosome with least difference gets selected. 


$$
\begin{array}{ll}
\mathrm{E}^{\mathrm{K}}{ }_{\text {Network- }} & \text { Current energy of network in } \mathrm{K}^{\text {th }} \text { round. } \\
\mathrm{E}^{\mathrm{k}-1} \text { Network- } & \text { Energy of network in previous i.e. } \mathrm{K}-1^{\text {th }} \\
\text { round } &
\end{array}
$$

\subsubsection{Algorithm}

- Network initialization.

- Position data of every sensor node is sent to neighbors.

- Fuzzy descriptors like energy, density and centrality are used to calculate a fuzzy parameter 'Chance'.

- Node who has higher 'Chance', a fuzzy parameter, than its neighbors will be a cluster head selection candidate.

- Now base station applies genetic algorithm based on chaotic and selects cluster heads.

- The network is informed about these new $\mathrm{CH}$.

- Nearest cluster heads are joined by remaining nodes.

Proposed method is successful in increasing lifetime of the network. Other parameter considered is number of messages received in base station. As the number increases, it prolongs network's lifetime.

\section{Advantages}

- Proposed method is efficient in prolonging lifetime of the network.

- Three types of nodes used are: normal node, advanced node and lowest energy node. Heterogeneous representation for dead nodes, nodes with low energy and cluster heads is used.

\section{Disadvantages}

- Here also the only criterion for calculating fitness is energy. Parameters like distance are not used.

- Computation of fitness function depends only upon the least energy difference as compared to last round.

\subsection{Method 4}

In paper [1], G. Ahmed proposed a genetic algorithm based cluster head selection. Four parameters are considered:

- Distance of the node from cluster centroid

- $\quad$ Remaining battery power

- Mobility

- Vulnerability

Three genetic operators used are: Reproduction, crossover and mutation.

Here a 4 bit binary number is used to represent a node. They have used X1X2X3X4

X1-Specifies the distance of the node from cluster centroid

X2-Vulnerability

X3-Degree of mobility

X4-Remaining battery power.
They have selected initial population randomly i.e population of size 4. Fitness function is function of different fitness parameters. Roulette wheel selection is used. Nodes which are able to copy itself in next generation are candidates for cluster head selection.

\section{Advantage}

- $\quad$ String which copies itself in next generation will become a cluster head.

- Efficient cluster heads are selected hence performance of the network increases.

\section{Disadvantages}

- There is some computational burden.

\subsection{Method 5}

In paper [2], Sudakshina Dasgupta presents a new approach of clustering in wireless sensor networks using genetic algorithm. Here a genetic algorithm is applied by base station. Basic idea is to evaluate the fitness of chromosomes. Depending upon the fitness value, next generation can be found that means chromosomes with higher fitness can be used to produce off springs for next generation. As a result of genetic algorithm, clusters are determined and the network details are broadcasted to all the nodes and clusters will be formed accordingly. In the proposed system:

- Binary representation is used for chromosomes i.e network is represented as bits of chromosomes.

- Roulette wheel selection is used where chromosomes with better fitness are used for offspring generation.

- One point crossover is used.

- Mutation is used.

Various fitness parameters used:

- Cluster distance

It is the distance from all sensor nodes plus distance from cluster head to base station.

- $\quad$ Transfer energy

Its total energy required to transmit a message from sensor nodes to cluster head plus energy required by $\mathrm{CH}$ to to receive messages from cluster members plus energy required to transmit aggregated message from $\mathrm{CH}$ to base station.

- Number of transmissions

Its decided by base station.

Fitness function as stated by [2]:

"Fitness $=(\mathrm{W} 1 * \mathrm{C}+\mathrm{W} 2 * \mathrm{E}+\mathrm{W} 3 * \mathrm{~T}) / \mathrm{E}_{0} "$

Here,

W1,W2, W3-Weight

$\mathrm{W} 1+\mathrm{W} 2+\mathrm{W} 3=1$

C-cluster distance

E-Transfer energy

T-Number of transmissions 


\section{$\mathrm{E}_{0}$-Initial energy of the node}

Simulation is done in Matlab. Performance is compared with LEACH. Proposed method was found more efficient than LEACH.

\section{Advantages}

- Proposed method is better in number of dead nodes after certain number of nodes.

- Proposed method increases energy reduction rate of the node.

- This method is the most efficient way of selecting $\mathrm{CH}$ and rotating them.

\section{Disadvantages}

- Certain parameters like crossover type, crossover rate, mutation rate are not changed. They are static.

- Computational overhead is involved.

\subsection{Method 6}

In paper [7], Shiuyan Jin proposed a genetic algorithm based clustering technique. Author has explained the scaling window concept. If individuals have similar fitness value then which one to select as cluster head becomes problematic. So to select a better individual as a cluster head, they have subtracted minimum fitness value from fitness value of each individual.

i.e. "fit(i)=fit(i)-fit ${ }_{\min } "$

They have used 2D environment with 100 nodes. They have tried two different sink positions $(0,0)$ and $(100,100)$. Value of certain parameters does not change throughout like population size, selection type, crossover rate, crossover type, mutation rate and generation size.

Fitness as specified by [7]:

"Fitness $=\mathrm{w}^{*}\left(\mathrm{D}-\right.$ distance $\left._{\mathrm{i}}\right)+(1-\mathrm{w}) *\left(\mathrm{~N}-\mathrm{H}_{\mathrm{i}}\right)$ "

Two scenarios are:

- When sink is at $(0,0)$

Here a weight is 1 so they have considered only the distance in fitness function.

- When sink is at $(200,200)$

$\mathrm{W}=0.8$

It was experimentally proved that this method is efficient in terms of energy saving.

\section{Advantages}

- If 100 nodes are considered a good solution is reached only after 120 generations.

- Nodes near to sink have more chances of becoming a cluster head.

- They have also shown that more number of cluster heads are required when sink is at center of network than when it is at corner.

- They have provided a solution when fitness values are closer. Solution is use of scaling window.

\section{Disadvantages}

- Crossover and mutation rate are static.

- As aim is to lower energy consumption, energy should be a fitness calculation parameter. Here energy is not considered

\subsection{Method 7}

In paper [3],Moslem Afrashteh Mehr proposed a method to determine optimum number of cluster heads and clustering. Basic aim was to reduce the energy consumption. Various genetic operators used are: crossover, selection, mutation and fitness.

Fitness function considers following parameters:

- Residual energy-This is main parameter for selection as aim is to reduce energy consumption.

- Energy required to send a message from $\mathrm{CH}$ to sink.

- D-Distance from sensors i.e. distance from all sensor nodes to sink minus distance from all sensor nodes to $\mathrm{CH}$ plus $\mathrm{CH}$ to sink.

Fitness function as stated by [3]:

"Fitness $=\mathrm{RE}+\mathrm{SE}+(\mathrm{X} *(\mathrm{D})+(1-\mathrm{X}) *(\mathrm{~N}-\mathrm{CH})) "$

$0<=\mathrm{X}<=1$

SE as stated by [3]:

$\mathrm{SE}=\sum_{\mathrm{i}=1}^{\mathrm{M}}\left(\mathrm{RES}_{\mathrm{i}}-\left(\mathrm{REC}_{\mathrm{i}}+(\mathrm{M}-1)(\mathrm{CHE}+\mathrm{CES})\right)\right.$

Where,

$\mathrm{RES}_{\mathrm{i}}$-Energy required to send a message from all the sensor nodes to sink.

$\mathrm{REC}_{\mathrm{i}}$ - Energy required to send a message from all the sensor nodes to $\mathrm{CH}$.

CHE-Total Energy required by $\mathrm{CH}$ to receive messages from all its cluster members

CES-Energy required to send a message from $\mathrm{CH}$ to sink.

Simulation is done in matlab. Proposed method when compared with classical LEACH was found more efficient.

\section{Advantages}

- Proposed method achieves uniform distribution of cluster heads.

- Energy consumption was found more uniform than LEACH.

- $\quad$ Proposed system has more number of alive nodes after certain number of rounds than LEACH.

\section{Disadvantages}

- Many parameters are considered in fitness function.

- $\quad$ Fitness function is complex.

- Computational overhead is involved. 


\section{COMPARATIVE STUDY}

Table 1.Comparative study of all the GA based clustering techniques

\begin{tabular}{|c|c|c|c|}
\hline $\begin{array}{r}\text { Name } \\
\text { of the method } \\
\end{array}$ & Fitness parameters & Advantages & Disadvantages \\
\hline Method 1 & $\begin{array}{l}\text { 1.Cluster distance } \mathrm{C} \text { ( It is } \\
\text { sum of distance from } \\
\text { member nodes to } \mathrm{CH} \text { and } \mathrm{CH} \\
\text { to BS) } \\
\text { 2.Round which first node } \\
\text { dies(Rfnd) } \\
\text { 3.Round which last node dies } \\
\text { (Rlnd) }\end{array}$ & $\begin{array}{l}\text { 1. Improves number of alive } \\
\text { nodes. } \\
\text { 2. Achieves energy efficiency. } \\
\text { 3. Improves network lifetime }\end{array}$ & 1. Energy is not a fitness parameter. \\
\hline Method 2 & $\begin{array}{l}\text { 1.N-Total number of nodes } \\
\text { 2.RE-Sum of residual energy } \\
\text { in cluster heads } \\
\text { 3.CH-Total number of cluster } \\
\text { heads } \\
\text { 4.X- Weight factor whose } \\
\text { value lies between } 0 \text { and } 1 \\
\text { 5. SE- Sum of the energy }\end{array}$ & $\begin{array}{l}\text { 1. Improves number of alive } \\
\text { nodes } \\
\text { 2. Achieves energy efficiency. } \\
\text { 3. Achieves uniform distribution } \\
\text { of the cluster heads }\end{array}$ & $\begin{array}{l}\text { 1. A single fitness parameter i.e. energy } \\
\text { is considered. }\end{array}$ \\
\hline Method 3 & $\begin{array}{l}\text { 1.EKNetwork-Current } \\
\text { energy of network in Kth } \\
\text { round. } \\
\text { 2.Ek-1Network- Energy of } \\
\text { network in previous i.e. K- } \\
\text { 1th round }\end{array}$ & 1.Achieves energy efficiency & $\begin{array}{l}\text { 1.Fitness calculation only considers } \\
\text { energy as a fitness parameter }\end{array}$ \\
\hline Method 4 & $\begin{array}{l}\text { 1. Distance of the node from } \\
\text { cluster centroid. } \\
\text { 2.Remaining battery power } \\
\text { 3. Mobility } \\
\text { 4. Vulnerability }\end{array}$ & $\begin{array}{l}\text { 1. Efficient cluster heads are } \\
\text { selected which helps in } \\
\text { enhancing lifetime of the } \\
\text { network. }\end{array}$ & 1. Computational overhead \\
\hline Method 5 & $\begin{array}{l}\text { 1. Cluster distance } \\
\text { 2. Transfer energy } \\
\text { 3. Number of transmissions }\end{array}$ & $\begin{array}{l}\text { 1. Better in number of dead } \\
\text { nodes } \\
2 . \text { Increases energy reduction } \\
\text { rate. } \\
3 \text {. Method is efficient way of } \\
\text { selecting } \mathrm{CH} \text { and rotating them }\end{array}$ & $\begin{array}{l}\text { 1. Computational overhead. } \\
\text { 2. Some parameters like crossover type, } \\
\text { crossover rate, and mutation rate are } \\
\text { static. }\end{array}$ \\
\hline Method 6 & $\begin{array}{l}\text { 1.D-Total distance from all } \\
\text { nodes t sink } \\
\text { 2.distance i- sum of the } \\
\text { distances from regular nodes } \\
\text { to cluster heads plus the sum } \\
\text { of the distances from all } \\
\text { cluster heads to the sink. } \\
\text { 3.w- weight factor } \\
\text { 4.N-number of sensor nodes } \\
\text { 5. Hi-Number of cluster } \\
\text { heads }\end{array}$ & $\begin{array}{l}\text { 1. Use of scaling windows when } \\
\text { fitness values are near. } \\
2 . \text { Reaches good solution only } \\
\text { after } 120 \text { generations. } \\
\text { 3. Nodes near sink have more } \\
\text { chance of getting selected as } \\
\text { cluster head. }\end{array}$ & $\begin{array}{l}\text { 1. Energy is not a fitness parameter. } \\
\text { 2. Crossover and mutation rates are } \\
\text { static. }\end{array}$ \\
\hline Method 7 & $\begin{array}{l}\text { 1. Residual energy-This is } \\
\text { main parameter for selection } \\
\text { as aim is to reduce energy } \\
\text { consumption. } \\
\text { 2. Energy required to send a } \\
\text { message from CH to sink. } \\
\text { 3. D-Distance from sensors } \\
\text { i.e. distance from all sensor } \\
\text { nodes to sink minus distance } \\
\text { from all sensor nodes to CH } \\
\text { plus CH to sink. }\end{array}$ & $\begin{array}{l}\text { 1. Achieves uniform distribution } \\
\text { of cluster heads } \\
2 \text {. Is better in number of alive } \\
\text { nodes. } \\
\text { 3. Consumption of energy is } \\
\text { uniform. }\end{array}$ & 1. More computational overhead. \\
\hline
\end{tabular}




\section{CONCLUSION}

One of the most important issues that should be addressed in order to improve life span of the network is efficiency of energy. Formation of clusters greatly helps in achieving energy efficiency as it reduces communication distance. Genetic algorithm ensures that fittest node is selected as cluster head. Paper discusses genetic algorithm based clustering technique along with their merits and demerits. Future work includes multi hop communication between cluster heads.

\section{REFERENCES}

[1] G. Ahmed, N. M. Khan, and R. Ramer,2008, "Cluster Head Selection Using Evolutionary Computing in Wireless Sensor Networks" .Progress In Electromagnetics Research Symposium, Hangzhou, China

[2] Sudakshina Dasgupta, Paramartha Dutta, "An energy efficient genetic approach for clustering of wireless sensor networks"

[3] Moslem Afrashteh Mehr, "Design and Implementation a New Energy Efficient Clustering Algorithm using Genetic Algorithm for Wireless Sensor Networks", World Academy of Science, Engineering and Technology 522011

[4] Selim Bayrakli,Senol Zafer Erdogan , "Genetic algorithm based energy efficient clusters(GABEEC) in wireless sensor networks", ScienceDirect Computer Networks 51 (2007) 1031-1051
[5] D.Srinivasa Rao, B.J.M. Ravi Kumar , "Performance Evaluation of Genetic Based Dynamic Clustering Algorithm over LEACH Algorithm for Wireless Sensor Networks", International Journal of Soft Computing and Engineering (IJSCE) ISSN: 2231-2307, Volume-1, Issue-4, September 2011

[6] Abbas Karimi, S. M. Abedini, Faraneh Zarafshan, S.A.R Al-Haddad, "Cluster Head Selection Using Fuzzy Logic and Chaotic Based Genetic Algorithm in Wireless Sensor Network", J. Basic. Appl. Sci. Res., 3(4)694-703, 2013

[7] Shiyuan Jin, Ming Zhou, Annie S. Wu, "Sensor network optimization using genetic algorithm"

[8] Sanjeev Wagh, Ramjee Prasad, "Heuristic Clustering for Wireless Sensor Networks using Genetic Approach", International Journal of Wireless and Mobile Networking (IJWAMN)Vol. 1, No. 1(November 2013)

[9] Jianming Zhang,Yaping Lin,Cuihong, Zhou,Jingcheng Ouyang, " Optimal Model for Energy-Efficient Clustering in Wireless Sensor Networks Using Global Simulated Annealing Genetic Algorithm", International Symposium on Intelligent Information Technology Application Workshops,2008.

[10] Basma Solaiman, Alaa Sheta, "Computational intelligence for wireless sensor networks: Applications and clustering algorithms, International Journal of Computer Applications (0975 - 8887) Volume 73 - No. 15 , July 2013 